\title{
Simulation of Early Calcific Aortic Valve Disease in a 3D platform: A Role for Myofibroblast Differentiation
}

Jesper Hjortnaes, $\mathrm{MD}^{1,2,3}$, Claudia Goettsch, $\mathrm{PhD}^{4}$, Joshua D. Hutcheson, $\mathrm{PhD},{ }^{4}$ Gulden CamciUnal, $\mathrm{PhD}^{2,5}$, Lilian Lax, MD ${ }^{1}$, Katrin Scherer, MD ${ }^{1}$, Simon Body, $\mathrm{MD}^{6}$, Frederick J. Schoen, $\mathrm{MD}, \mathrm{PhD}^{7}$, Jolanda Kluin, $\mathrm{MD}, \mathrm{PhD}^{3}$, Ali Khademhosseini, $\mathrm{PhD}^{2,5,8 *}$, Elena Aikawa, $\mathrm{MD}$, $\mathrm{PhD}^{1,4 *}$

\section{Affiliations}

${ }^{1}$ Center of Excellence in Vascular Biology, Department of Medicine, Brigham and Women's Hospital, Harvard Medical School, Boston, MA, USA; ${ }^{2}$ Center for Biomedical Engineering, Department of Medicine, Brigham and Women's Hospital, Harvard Medical School, Boston, MA, USA; ${ }^{3}$ Department of Cardiothoracic Surgery, University Medical Center Utrecht, Utrecht, The Netherlands; ${ }^{4}$ Center for Interdisciplinary Cardiovascular Sciences, Department of Medicine, Brigham and Women's Hospital, Boston, MA, USA; ${ }^{5}$ Harvard-MIT Division of Health Sciences and Technology, Massachusetts Institute of Technology, Cambridge, MA, USA;

${ }^{6}$ Department of Anesthesiology, Perioperative and Pain Medicine, Brigham and Women's Hospital, Harvard Medical School, Boston; ${ }^{7}$ Department of Pathology, Brigham and Women's Hospital, Harvard Medical School, Boston, MA, USA; ${ }^{8}$ Wyss Institute for Biologically Inspired Engineering, Harvard University, Boston, MA, USA

Word count: 5892 (including Methods)

*Correspondence:

Elena Aikawa, M.D., Ph.D.

Brigham and Women's Hospital, Harvard Medical School

77 Avenue Louis Pasteur, Boston, MA 02115, USA

Tel: $617-730-7755$

Email: eaikawa@partners.org or alik@bwh.harvard.edu 


\begin{abstract}
Purpose: Calcific aortic valve disease (CAVD) is the most prevalent valve disease in the Western world. Recent difficulty in translating experimental results on statins to beneficial clinical effects warrants the need for understanding the role of valvular interstitial cells (VICs) in CAVD. In two-dimensional culture conditions, VICs undergo spontaneous activation similar to pathological differentiation, which intrinsically limits the use of in vitro models to study CAVD. Here, we hypothesized that a three-dimensional (3D) culture system based on naturally derived extracellular matrix polymers, mimicking the microenvironment of native valve tissue, could serve as a physiologically relevant platform to study the osteogenic differentiation of VICs.

Principal results: Aortic VICs loaded into 3D hydrogel constructs maintained a quiescent phenotype, similar to healthy human valves. In contrast, osteogenic environment induced an initial myofibroblast differentiation (hallmarked by increased alpha smooth muscle actin $[\alpha-$ SMA] expression), followed by an osteoblastic differentiation, characterized by elevated Runx 2 expression, and subsequent calcific nodule formation recapitulating CAVD conditions. Silencing of $\alpha$-SMA under osteogenic conditions diminished VIC osteoblast-like differentiation and calcification, indicating that a VIC myofibroblast-like phenotype may precede osteogenic differentiation in CAVD.
\end{abstract}

Major conclusions: Using a 3D hydrogel model, we simulated events that occur during early CAVD in vivo and provided a platform to investigate mechanisms of CAVD. Differentiation of valvular interstitial cells to myofibroblasts was a key mechanistic step in the process of early mineralization. This novel approach can provide important insight into valve pathobiology and serve as a promising tool for drug screening.

Keywords: Aortic valve stenosis, calcific aortic valve disease, tissue engineering, hydrogels, valvular interstitial cells 


\section{Introduction}

Aortic valve stenosis due to calcific aortic valve disease (CAVD) is the most common heart valve disease in developed countries. ${ }^{1}$ CAVD is a progressive disease characterized by a cascade of cellular events in the valve leaflets that results in fibrotic leaflet thickening and calcification, impairing functional leaflet movement and causing left ventricular outflow obstruction. ${ }^{2}$

Although CAVD traditionally was viewed as a passive degenerative phenomenon resulting from years of mechanical stress, we now recognize it as an actively regulated disease, with evidence suggesting a process akin to bone formation. ${ }^{3,4}$ The disease progression is primarily regulated by valve interstitial cells (VICs), the most abundant cell type in the aortic valve. End-point analyses of calcified leaflets have identified various VIC phenotypes, including quiescent fibroblast-like VICs, which can differentiate into activated myofibroblast-like VICs during various pathological conditions, but also have revealed the presence of osteoblast-like cells which may be responsible for the deposition of calcium mineral in CAVD. ${ }^{3,5-8}$ VIC differentiation accelerates in response to pathophysiological cues caused by injury or disease, ${ }^{9}$ which include inflammation ${ }^{10,11}$, oxidized low-density lipoprotein (LDL) deposits ${ }^{12,13}$, oxidative stress ${ }^{14}$, and extracellular matrix (ECM) disruption. ${ }^{15,16}$ Understanding pathological changes in VICs is hindered by a lack of in vitro model systems that maintain physiological VIC phenotypes without spontaneous VIC myofibroblast-like differentiation, as is observed when cultured on unnaturally stiff tissue culture plates. No in vitro study, however, has identified all of the relevant VIC phenotypes and their respective contributions to CAVD. ${ }^{15,17}$ Myofibroblast-like VICs form dystrophic calcific nodules in vitro; however, these nodules do not display osteogenic attributes ${ }^{15,18}$ and osteoblast-like differentiation of VICs in vitro has proven difficult. ${ }^{19}$ Given that both dystrophic and osteogenic calcification processes are observed in excised human CAVD leaflets, ${ }^{3,20}$ in vitro platforms that enable the study of both myofibroblast-like and osteoblast-like VICs are needed.

Three-dimensional (3D) culture platforms are emerging to provide a more tissue-like environment for studying valvular cell behavior. ${ }^{17,21}$ Such 3D cell culture systems could simulate the natural ECM of the valve and model an in situ-like environment of the fibrosa layer, where calcification is mainly observed. ${ }^{22}$ Photocrosslinkable hydrogels used to engineer 3D culture platforms for VICs showed limited damage of encapsulated cells during fabrication ${ }^{17,21 \text {, }}$

${ }^{23}$ and preservation of cell-matrix interaction and matrix signaling. ${ }^{24}$ Hyaluronic acid, an important glycosaminoglycan of the adult heart valve ECM, is a vital component of the cardiac 
jelly during heart embryogenesis. Gelatin — a denatured form of collagen - is abundantly present in the heart valve ECM. By adding methacrylate groups to hyaluronic acid (HAMA) and gelatin (GelMA), these biopolymers can crosslink to each other in the presence of a photoinitiator and form 3D hydrogel platforms. ${ }^{25,26,27} \mathrm{We}$ have recently shown that combining HAMA and GelMA into a 3D hybrid hydrogel platform can maintain a quiescent VIC phenotype, while allowing VICs to undergo myofibroblast-like differentiation upon exogenous pathological stimulation. ${ }^{30}$ This study provided us with an in vitro 3D platform that can be used to model VIC phenotype changes as thought to occur in early CAVD. In this study, we use a controllable in vitro 3D model system to elucidate the relationship between myofibroblast-like and osteoblast-like VIC phenotypes in CAVD.

\section{Methods}

For detailed methods see supplemental material.

\section{1 Valvular interstitial cell (VIC) isolation and culture}

Porcine VICs were isolated from aortic heart valves as described previously ${ }^{28}$ and cultured in normal growth medium containing high glucose (4.5g/l) Dulbecco's modified Eagle's medium (DMEM with $10 \%$ fetal bovine serum and $1 \%$ penicillin/streptomycin (Invitrogen)) at $37^{\circ} \mathrm{C}, 5 \%$ $\mathrm{CO}_{2}$. Cells between passage 3 and 6 were used for all experiments.

\subsection{Human tissue}

Human calcified aortic valves were obtained from CAVD patients who underwent surgical valve replacement according to Brigham and Women's Hospital Institutional Review Board Protocol (2010P002567/BWH). Samples were embedded in optimal cutting temperature compound (OCT) and stored at $-80^{\circ} \mathrm{C}$ until use.

\subsection{Hydrogel fabrication}

Hybrid hydrogels were fabricated from hyaluronic acid (HAMA) and gelatin (GelMA), which were synthesized as reported previously ${ }^{29,25,30}$ using photocrosslinking (Figure 1). ${ }^{26,28}$ Briefly, VICs were resuspended in the prepolymer solution consisting of $1 \mathrm{wt} \%$ HAMA and $5 \mathrm{wt} \%$

GelMA. ${ }^{25} 50 \mu \mathrm{L}$ of the cell-laden polymer solution was added between two spacers each with a 
height of $450 \mu \mathrm{m}$ and subjected to light (wavelength $360 \mathrm{~nm}$ ) with a light intensity of 2.5 $\mathrm{mW} / \mathrm{cm}^{2}$. VIC-laden hydrogels were cultured in either control medium (CM) or osteogenic medium (OM) (CM supplemented with $10 \mathrm{mM} \beta$-glycerophosphate, $10 \mathrm{ng} / \mathrm{mL}$ ascorbic acid, and $10 \mathrm{nM}$ dexamethasone) or OM with tumor necrosis factor alpha (TNF $\alpha$ ).

\subsection{Silencing of alpha smooth muscle actin in VIC-laden hydrogels}

To inhibit the expression of $\alpha$-SMA, ON-TARGET plus SMART-pool (L-003605-00-00100) and a negative control (scramble) from Thermo Scientific was used. Transfection of $50 \mathrm{nmol} / \mathrm{l}$ siRNAs was performed using DharmaFECT 1 transfection reagent twice per week over the entire culture period.

\subsection{Histological analysis and immunofluorescence staining of human valve and VIC-laden} hydrogel constructs

VIC-laden hydrogels were frozen in optimal cutting temperature (OCT) compound, and 10- $\mu \mathrm{m}$ cross-sections were obtained. To detect calcium deposition, sections were stained with 0.02 mg/mL Alizarin Red S solution (Sigma). Von Kossa staining (American MasterTech) was used to visualize calcium-phosphate deposition.

Immunofluorescence staining for $\alpha$-SMA and runt-related transcription factor 2 (Runx2) was performed using anti-human $\alpha$-SMA antibody (Clone 1A4, Dako) or an anti-human Runx2 antibody (Abcam), followed by biotin-labeled secondary antibody (Vector Labs) and streptavidin-labeled AlexaFluor 488 for $\alpha$-SMA and AlexaFluor 594 for Runx2. Sections were counterstained with 4',6-diamidino-2-phenylindole (DAPI). Images were taken with an Eclipse 80i microscope (Nikon) and processed with Elements 3.20 software (Nikon). A custom MATLAB-based script was used to detect DAPI-stained cellular nuclei in representative images of calcified valves from four different human donors. Quantification of immunofluorescent signal surrounding and within each nucleus provided information on $\alpha$-SMA expression and Runx 2 expression and nuclear translocation on a cell-by-cell basis.

\subsection{Quantification of alkaline phosphatase activity and calcium content in hydrogel constructs} Activity of alkaline phosphatase (ALP) and calcium content were measured using colorimetric assays (Biovision Lifesciences) and normalized to DNA content. 
2.7 Real-Time Polymerase Chain Reaction for expression of cell markers

After mechanical disruption of the cell-laden hydrogels (TissueLyzer, Qiagen), total RNA was isolated using RNAspin mini RNA Isolation kit (GE Healthcare). Total RNA was reverse transcribed with oligo-(dT)12-18 primers and Superscript II reverse transcriptase (Life Technologies) followed by RT- PCR using SYBR Green (BioRad). Primer sequences were as follows: $\alpha$-SMA: F:5'-AGTGCGACATTGACATCAGG-‘3 and R:5'-

CTGGAAGGTGGACAGAGAGG-‘3, Runx2 F:5’-ACCCAGAAGACTGTGGATGG-‘3 and R:5’-ACCTGGTCCTCAGTGTAGCC-`3, GAPDH: F:5'-CCCAGAAGACTGTGGATGG-‘3 , R:5'-ACCTGGTCCTCAGTGTAGCC-`3. Expression was quantified using comparative $2^{-\Delta \Delta C T}$ method.

\subsection{Cell viability and apoptosis}

Cell viability was determined by LIVE/DEAD Viability kit (Life Technologies). Apoptosis was determined by TUNEL staining (Millipore). Cell-laden hydrogels were imaged using an A1/C1 confocal microscope (Nikon).

\subsection{Statistical analysis}

Results are presented as mean $+/$ - standard deviation unless indicated otherwise. Unpaired student's t-test was used for comparisons between two groups. Two-way ANOVA was used to evaluate statistical significant differences in multiple groups over time. $\mathrm{P}<0.05$ was considered statistically significant.

\section{Results}

\subsection{An osteogenic environment activates quiescent VICs and induces calcific nodule formation in $3 D$ culture constructs}

We previously showed that VICs cultured in 3D hydrogels maintained a quiescent phenotype similar to that observed in normal aortic valve leaflets, whereas 2D culture either on hydrogels or tissue culture plastic led to spontaneous activation to a pathological myofibroblast-like 
phenotype $\mathrm{e}^{28}$. Therefore, we proposed that the 3D hydrogel system is more suited to study calcific nodule formation by VICs in response to pathologic stimuli. To evaluate the role of inflammation as pathophysiology related to early $\mathrm{CAVD}^{11}$, we also used the pro-inflammatory cytokine $\mathrm{TNF}^{31,32}$

VICs cultured in an osteogenic 3D environment stimulated calcific nodule formation visualized by Alizarin Red S staining. Calcific nodules localized throughout the VIC-laden hydrogel and particularly increased at day 21 (Figure 2A). VIC stimulation with osteogenic media (OM) and $\mathrm{TNF} \alpha$ promoted calcific nodule formation visualized by Alizarin Red S (Figure 2B) and von Kossa staining (Figure 2C, Supplemental Figure I), while TNFa supplemented to control media (CM) had no effect. Quantification of nodule formation revealed a 1.65-fold increase in VICladen hydrogels cultured in OM supplemented with TNF $\alpha$ compared to untreated cells in 3D ( $p$ $<0.05$, Figure 2D). Quantitative calcium analysis showed a time-dependent OM-mediated induction of calcium deposition that was further increased by $\mathrm{TNF} \alpha$ stimulation (Figure 2E). ALP activity, a marker of early calcification, peaked at day 10 (Supplemental Figure II). Addition of TNFa to OM significantly increased ALP activity (OM: 1.2 \pm 0.1 vs. OM+TNFa: $2.3 \pm 0.9, \mathrm{p}<0.05)$. 3D cultures in CM showed no ALP activity (data not shown).

Cell viability in the hydrogels was $95 \pm 2 \%$ and $91 \pm 2 \%$ at day 1 and $90 \% \pm 2$ and $87 \pm 1 \%$ at day 21 in CM and OM culture, respectively (Supplemental Figure III). TUNEL staining and quantification showed no apoptosis at 7, 14, and 21 days of culture in constructs cultured in OM and OM+TNFa (Supplemental Figure IV). These results suggest that apoptosis does not play a major role in mineralization within VIC-laden hydrogels, and that TNF $\alpha$ exacerbates mineralization in the $3 \mathrm{D}$ constructs.

Next, we studied the relationship of myofibroblast-like and osteoblast-like differentiation of quiescent VICs in calcific conditions. We compared $\alpha$-SMA expression of VICs in human valves and VICs cultured in the 3D hydrogels in CM or OM.

Non-calcified areas of non-diseased regions of human aortic valves expressed low $\alpha$-SMA levels (Figure 3A, B), while calcified areas acquire increased $\alpha$-SMA levels (Figure 3C, D). VICs laden in 3D hydrogels cultured in OM showed similar increase in $\alpha$-SMA levels compared to CM (Figure 3E, F). Quantitative analysis revealed a 2-fold increase in $\alpha$-SMA-positive cells within the VIC-laden hydrogels (Figure 3G) and a significant increase in $\alpha$-SMA mRNA expression 
(Figure 3H) after culturing VICs for 21 days in OM compared to CM, indicating an activated myofibroblast-like VIC phenotype.

A time course study of gene expression in the hydrogels revealed that $\alpha$-SMA mRNA expression peaked at day 7 and exhibited a time-dependent decrease at days 14 and 21 for both OM and $\mathrm{OM}+\mathrm{TNF} \alpha$ (Figure 3I, two-way ANOVA from day 7 to 21, $\mathrm{p}=0.001$ ). Immunofluorescence for $\alpha$-SMA also revealed a peak in the percentage of myofibroblast-like VICs in the hydrogels at day 7 for both Om and OM+TNF $\alpha$ treatments (Supplemental Figure V). Runx2, a transcription factor for osteoblast-like differentiation, significantly increased on mRNA level in both OM and $\mathrm{OM}+\mathrm{TNF} \alpha$ after day 7 (Figure 3J, two-way ANOVA from day 7 to 21, $\mathrm{p}=0.05$ ). These results suggest that myofibroblast-like differentiation may precede osteoblastic differentiation of quiescent VICs when cultured in an osteogenic environment. Within the 3D hydrogels cultured in OM for 21 days, we observed three distinct VIC phenotypes immuno-positive for Runx2: 1) VICs that exhibit both $\alpha$-SMA and cytosolic Runx2 expression (Figure 3K); 2) VICs that exhibit cytosolic Runx2 expression but no $\alpha$-SMA expression (Figure 3L); and 3) VICs that exhibit nuclear translocation of Runx2 expression and no $\alpha$-SMA expression (Figure 3M).

\subsection{Reduction of a-SMA expression diminishes VIC mineralization in $3 D$ constructs}

Since we observed an initial increase in $\alpha$-SMA expression followed by Runx 2 expression (Figure 3I-J), we next investigated a causal relationship between VIC myofibroblast-like differentiation, VIC osteoblast-like differentiation, and calcification. VIC-laden hydrogels were cultured in CM, OM, and OM+TNF $\alpha$ under the silencing of $\alpha$-SMA by siRNA or scrambled non-targeted siRNA (SCR) as control. Immunofluorescence staining for $\alpha$-SMA (Supplemental Figure VI) and analysis of mRNA expression levels (Figure 4A) demonstrated $80 \%$ silencing efficiency. $\alpha$-SMA silencing reduced Runx2 (Figure 4B) expression and ALP activity (Supplemental Figure VII A, B) in OM and OM+TNF $\alpha$ cultures compared to control. Concomitantly, $\alpha$-SMA silencing caused a significant reduction in calcification, as detected by quantitative calcium analysis (Figure 4C), Alizarin Red S staining (Figure 4D), and von Kossa staining (Supplemental Figure VIII), suggesting that myofibroblast-like activation is required for VIC osteogenic transformation and calcification within the 3D hydrogels.

\subsection{Association and quantification of VIC phenotypes in human CAVD}


We next assessed the association between myofibroblast-like VICs and osteoblast-like VICs in human CAVD tissues. At least three representative high power images were collected and analyzed from histological sections of leaflets from four different human donors (Supplemental Figure IX). Immunofluorescence staining for Runx2 and $\alpha$-SMA (Figure 5A) showed regions positive for both phenotypic markers. A custom MATLAB-based script quantified $\alpha$-SMA immunofluorescence and Runx2 immunofluorescence on a cell-by-cell basis (Figure 5B), and allowed analysis of over 24,000 cells from the four human donor tissues. VICs with high $\alpha$-SMA expression exhibited low Runx2 expression and VICs with high Runx 2 expression exhibited low $\alpha$-SMA expression. VICs with moderate expression of both markers were also observed. Quantification of the fraction of Runx2 within the nucleus of each cell revealed that VICs with Runx2 nuclear translocation almost exclusively exhibited low $\alpha$-SMA expression (Figure 5C). From this plot, we observed four pathologic VIC phenotypes in human CAVD tissues similar to the phenotypes observed in the hydrogel model (Section 3.2): 1) VICs with high $\alpha$-SMA expression but low Runx2 expression (Figure 5D); 2) VICs with moderate $\alpha$-SMA expression and Runx2 expression (Figure 5E); 3) VICs with high cytosolic Runx2 expression and low $\alpha$ SMA expression (Figure 5F); 4) VICs with Runx2 nuclear translocation and no observable $\alpha$ SMA expression (Figure 5G).

\section{Discussion}

This study establishes an in vitro 3D culture model to study VIC differentiation phenotypic patterns in CAVD (Figure 6). We previously showed that the 3D hybrid hydrogel platform composed of hyaluronic acid and gelatin maintains a quiescent VIC phenotype identified in healthy heart valves, thus providing a platform to study phenotypic changes associated with CAVD. ${ }^{28}$ Quiescent VICs in the hydrogel were activated by osteogenic stimuli to undergo myofibroblast-like differentiation and eventually differentiate into osteoblast-like cells, providing a platform that allows observation of both myofibroblast-like and osteoblast-like VICs - the two pathologic phenotypes observed in CAVD. Furthermore, we established that myofibroblast-like differentiation, hallmarked by $\alpha$-SMA expression, is necessary for osteoblastlike differentiation, and the observed phenotypes in the hydrogel model (Figure 4) seem to mirror those observed in human CAVD tissues (Figure 5). We showed that this in vitro 3D 
model could be used to controllably study specific pathological mechanisms associated with CAVD, including inflammation and calcification.

Recent evidence suggests that early CAVD may be characterized as an active disease process, in which quiescent VICs become activated to undergo myofibroblast-like differentiation upon exposure to mechanical stress or inflammation, hallmarked by the presence of $\alpha-S M A$ stress-fibers within the cytoskeleton. ${ }^{4}$ Activated VICs maintain valvular tissue integrity by pertinent remodeling of the valve ECM, by secreting cytokines, ${ }^{8,33}$ matrix metalloproteinases, ${ }^{6,}, 34$ and depositing ECM proteins. ${ }^{33,35}$ In addition, osteoblastic cells have been detected in the surgically excised heart valves and animal models, ${ }^{3,5}$ characterized by Runx 2 expression. ${ }^{11}$ Causal relationships between VIC phenotypes, however, have not been established. The present study is the first, to our knowledge, reporting that quiescent VICs differentiate into osteoblastlike VICs by first undergoing myofibroblast differentiation. This may explain the observation of both dystrophic and osteogenic calcific deposits in vivo. Dystrophic nodules may form directly by apoptotic myofibroblast-like VICs, ${ }^{33,36}$ but certain VIC subpopulation may proceed to osteogenic nodules through myofibroblast activation preceding an osteoblast-like phenotype.

Growing evidence, supported by our data, suggests that studying VIC pathophysiology in vitro is highly dependent on the culture microenvironment. ${ }^{15,37}{ }^{28}$ More specifically, matrix elasticity and cell-matrix protein interactions play important roles in directing the pathological response of VICs. ${ }^{15}$ VICs cultured on standard tissue culture polystyrene, an unnaturally stiff environment, undergo a complete phenotypic change into myofibroblast-like cells ${ }^{38}$ - commonly considered a "diseased" state. Hence, numerous studies have focused on modulating 2D culture substrates, albeit by changing stiffness or by using various ECM proteins, to analyze their relationship to VIC biology. ${ }^{37-39}$ Stiffer substrates elicit increased myofibroblast-like differentiation and even calcific nodule formation; ${ }^{15,38}$ however, osteoblastic markers were not identified in these studies. Indeed, when cultured on stiff gels ( $>25 \mathrm{kPa})$, VICs seemed to form larger calcific nodules with apoptotic cells, ${ }^{15}$ similar to observations in dystrophic calcification. ${ }^{9}$ As opposed to 2D culture, ${ }^{15,39}$ the myofibroblast-like response of VICs encapsulated into 3D hydrogels only occurs when exposed to exogenous environmental cues. The elastic modulus of 3D hydrogels used in our study $\left(\sim 20 \mathrm{kPA}{ }^{28}\right)$ corresponds to the perceived modulus of the fibrosa as measured by micropipette aspiration up to $21 \mathrm{kPA} .{ }^{40}$ Notably, mineralization of the aortic 
valve by VICs predominantly occurs in the fibrosa of the heart valve ECM, ${ }^{9}$ which recently was shown to have a relatively soft modulus. ${ }^{40}$

In the present study, we did not observe apoptotic driven calcification in 3D VIC-laden hydrogels, but rather an "active" osteogenesis via the Runx2 pathway, in which calcific nodules, composed of calcium and phosphates, are formed throughout the construct. On a cellular level, we observed the ability of healthy quiescent VICs to differentiate into osteoblast-like VICs - a process that, in the 3D environment, requires initial myofibroblast-like activation and reorganization of the cytoskeleton demonstrated by expression of $\alpha$-SMA. Our observations offer insight into a lingering question in VIC biology: do osteoblast-like VICs solely derive from resident quiescent VICs or does a heterogeneous VIC population contain specific mesenchymal or osteogenic progenitor cell subpopulation ${ }^{37}$ Considering that myofibroblast-like differentiation may be required for VIC transition this could pose more specific pathways for possible therapeutic intervention. Our study is limited by the static nature of 3D hydrogel platform and the absence of an endothelial monolayer, both important components when modeling valvular biology. Blood flow-induced shear stress and hemodynamic forces are key in valve tissue development, structural integrity, and disease. ${ }^{9}$ As is apparent from valve development and tissue engineering, ${ }^{41,42}$ biomechanical signals that are transduced to interaction between valvular endothelial cells, VICs, and the ECM are vital to understanding valve biology and pathology. ${ }^{43-45}$ Further, we attempted to model aspects of inflammation in this study by adding TNF $\alpha$ to the hydrogels. While our data show that this cytokine exacerbates VIC calcification, inflammatory cells play a larger role in CAVD than solely the release of TNF $\alpha$ (e.g., elaboration of proteases and other cytokines). Future studies could use this platform as a means to test co-culture of VICs with inflammatory cells to more fully address the scope of inflammation-driven CAVD.

In conclusion, our study simulates events that occur in early CAVD using a 3D hydrogel culture platform, in which quiescent VICs differentiate to osteoblast-like cells through initial myofibroblast-like differentiation. The 3D approach presented in this work can maintain healthy quiescent VIC population and thus can model the entire cellular process. This platform may provide a tool for drug screening and for the validation of cellular and molecular mechanisms in CAVD. 


\section{Acknowledgements}

The authors acknowledge Sara Karwacki for her excellent editorial assistance.

\section{Funding}

This work was supported by the Dutch Heart Foundation (DHF) and Netherlands Scientific

Council (NWO) (to J.H.), the Office of Naval Research Young National Investigator Award, the Presidential Early Career Award for Scientists and Engineers (PECASE), the National Science Foundation CAREER Award (DMR 0847287), and the National Institutes of Health (EB012597, HL092836, DE019024, HL099073, AR057837, EB008392, DE021468, to A.K.; NIH R01 HL114805 and NIH R01 HL109506, to E.A.). 


\section{References}

1. Stewart BF, Siscovick D, Lind BK, Gardin JM, Gottdiener JS, Smith VE, Kitzman DW and Otto CM. Clinical factors associated with calcific aortic valve disease. Cardiovascular Health Study. J Am Coll Cardiol. 1997;29:630-4.

2. Otto CM. Calcific aortic stenosis--time to look more closely at the valve. $N$ Engl J Med. 2008;359:1395-8.

3. Mohler ER, 3rd, Gannon F, Reynolds C, Zimmerman R, Keane MG and Kaplan FS. Bone formation and inflammation in cardiac valves. Circulation. 2001;103:1522-8.

4. Yutzey KE, Demer LL, Body SC, Huggins GS, Towler DA, Giachelli CM, Hofmann-Bowman MA, Mortlock DP, Rogers MB, Sadeghi MM and Aikawa E. Calcific aortic valve disease: a consensus summary from the Alliance of Investigators on Calcific Aortic Valve Disease. Arteriosclerosis, thrombosis, and vascular biology. 2014;34:2387-93.

5. Rajamannan NM, Subramaniam M, Rickard D, Stock SR, Donovan J, Springett M, Orszulak T, Fullerton DA, Tajik AJ, Bonow RO and Spelsberg T. Human aortic valve calcification is associated with an osteoblast phenotype. Circulation. 2003;107:2181-4.

6. Rabkin-Aikawa E, Farber M, Aikawa M and Schoen FJ. Dynamic and reversible changes of interstitial cell phenotype during remodeling of cardiac valves. J Heart Valve Dis. 2004;13:841-7.

7. Mohler ER, 3rd, Chawla MK, Chang AW, Vyavahare N, Levy RJ, Graham L and Gannon FH. Identification and characterization of calcifying valve cells from human and canine aortic valves. $J$ Heart Valve Dis. 1999;8:254-60.

8. Chester AH and Taylor PM. Molecular and functional characteristics of heart-valve interstitial cells. Philosophical transactions of the Royal Society of London Series B, Biological sciences. 2007;362:1437-43.

9. Schoen FJ. Evolving concepts of cardiac valve dynamics: the continuum of development, functional structure, pathobiology, and tissue engineering. Circulation. 2008;118:1864-80.

10. Hjortnaes J, Butcher J, Figueiredo JL, Riccio M, Kohler RH, Kozloff KM, Weissleder R and Aikawa E. Arterial and aortic valve calcification inversely correlates with osteoporotic bone remodelling: a role for inflammation. Eur Heart J. 2010;31:1975-84.

11. Aikawa E, Nahrendorf M, Sosnovik D, Lok VM, Jaffer FA, Aikawa M and Weissleder R. Multimodality molecular imaging identifies proteolytic and osteogenic activities in early aortic valve disease. Circulation. 2007;115:377-86.

12. Rajamannan NM, Subramaniam M, Caira F, Stock SR and Spelsberg TC. Atorvastatin inhibits hypercholesterolemia-induced calcification in the aortic valves via the Lrp5 receptor pathway. Circulation. 2005;112:1229-34.

13. Rajamannan NM. The role of Lrp5/6 in cardiac valve disease: LDL-density-pressure theory. Journal of cellular biochemistry. 2011;112:2222-9.

14. Miller JD, Chu Y, Brooks RM, Richenbacher WE, Pena-Silva R and Heistad DD. Dysregulation of antioxidant mechanisms contributes to increased oxidative stress in calcific aortic valvular stenosis in humans. J Am Coll Cardiol. 2008;52:843-50.

15. Yip $\mathrm{CY}, \mathrm{Chen} \mathrm{JH}$, Zhao $\mathrm{R}$ and Simmons $\mathrm{CA}$. Calcification by valve interstitial cells is regulated by the stiffness of the extracellular matrix. Arteriosclerosis, thrombosis, and vascular biology. 2009;29:93642.

16. Schoen FJ, Tsao JW and Levy RJ. Calcification of bovine pericardium used in cardiac valve bioprostheses. Implications for the mechanisms of bioprosthetic tissue mineralization. The American journal of pathology. 1986;123:134-45. 
17. Benton JA, Fairbanks BD and Anseth KS. Characterization of valvular interstitial cell function in three dimensional matrix metalloproteinase degradable PEG hydrogels. Biomaterials. 2009;30:6593-603.

18. Hutcheson JD, Aikawa E and Merryman WD. Potential drug targets for calcific aortic valve disease. Nature reviews Cardiology. 2014;11:218-31.

19. Monzack EL and Masters KS. Can valvular interstitial cells become true osteoblasts? A side-byside comparison. J Heart Valve Dis. 2011;20:449-63.

20. Torre M, Hwang DH, Padera RF, Mitchell RN and VanderLaan PA. Osseous and chondromatous metaplasia in calcific aortic valve stenosis. Cardiovascular pathology : the official journal of the Society for Cardiovascular Pathology. 2016;25:18-24.

21. Masters KS, Shah DN, Walker G, Leinwand LA and Anseth KS. Designing scaffolds for valvular interstitial cells: cell adhesion and function on naturally derived materials. Journal of biomedical materials research Part A. 2004;71:172-80.

22. Otto CM, Kuusisto J, Reichenbach DD, Gown AM and O'Brien KD. Characterization of the early lesion of 'degenerative' valvular aortic stenosis. Histological and immunohistochemical studies. Circulation. 1994;90:844-53.

23. Masters KS, Shah DN, Leinwand LA and Anseth KS. Crosslinked hyaluronan scaffolds as a biologically active carrier for valvular interstitial cells. Biomaterials. 2005;26:2517-25.

24. Slaughter BV, Khurshid SS, Fisher OZ, Khademhosseini A and Peppas NA. Hydrogels in regenerative medicine. Adv Mater. 2009;21:3307-29.

25. Nichol JW, Koshy ST, Bae H, Hwang CM, Yamanlar S and Khademhosseini A. Cell-laden microengineered gelatin methacrylate hydrogels. Biomaterials. 2010;31:5536-44.

26. Camci-Unal G, Cuttica D, Annabi N, Demarchi D and Khademhosseini A. Synthesis and characterization of hybrid hyaluronic acid-gelatin hydrogels. Biomacromolecules. 2013;14:1085-92.

27. Shah DN, Recktenwall-Work SM and Anseth KS. The effect of bioactive hydrogels on the secretion of extracellular matrix molecules by valvular interstitial cells. Biomaterials. 2008;29:2060-72.

28. Hjortnaes J, Camci-Unal G, Hutcheson JD, Jung SM, Schoen FJ, Kluin J, Aikawa E and Khademhosseini A. Directing valvular interstitial cell myofibroblast-like differentiation in a hybrid hydrogel platform. Advanced healthcare materials. 2015;4:121-30.

29. Camci-Unal G, Nichol JW, Bae H, Tekin H, Bischoff J and Khademhosseini A. Hydrogel surfaces to promote attachment and spreading of endothelial progenitor cells. Journal of tissue engineering and regenerative medicine. 2012.

30. Ramon-Azcon J, Ahadian S, Obregon R, Camci-Unal G, Ostrovidov S, Hosseini V, Kaji H, Ino K, Shiku H, Khademhosseini A and Matsue T. Gelatin methacrylate as a promising hydrogel for 3D microscale organization and proliferation of dielectrophoretically patterned cells. Lab on a chip. 2012;12:2959-69.

31. Al-Aly Z, Shao JS, Lai CF, Huang E, Cai J, Behrmann A, Cheng SL and Towler DA. Aortic Msx2-Wnt calcification cascade is regulated by TNF-alpha-dependent signals in diabetic Ldlr-/- mice.

Arteriosclerosis, thrombosis, and vascular biology. 2007;27:2589-96.

32. Tintut $\mathrm{Y}$, Patel J, Parhami F and Demer LL. Tumor necrosis factor-alpha promotes in vitro calcification of vascular cells via the cAMP pathway. Circulation. 2000;102:2636-42.

33. Walker GA, Masters KS, Shah DN, Anseth KS and Leinwand LA. Valvular myofibroblast activation by transforming growth factor-beta: implications for pathological extracellular matrix remodeling in heart valve disease. Circ Res. 2004;95:253-60.

34. Rabkin E, Aikawa M, Stone JR, Fukumoto Y, Libby P and Schoen FJ. Activated interstitial myofibroblasts express catabolic enzymes and mediate matrix remodeling in myxomatous heart valves. Circulation. 2001;104:2525-32. 
35. Cushing MC, Liao JT and Anseth KS. Activation of valvular interstitial cells is mediated by transforming growth factor-beta1 interactions with matrix molecules. Matrix biology : journal of the International Society for Matrix Biology. 2005;24:428-37.

36. Hutcheson JD, Chen J, Sewell-Loftin MK, Ryzhova LM, Fisher Cl, Su YR and Merryman WD. Cadherin-11 regulates cell-cell tension necessary for calcific nodule formation by valvular myofibroblasts. Arteriosclerosis, thrombosis, and vascular biology. 2013;33:114-20.

37. Chen JH and Simmons CA. Cell-matrix interactions in the pathobiology of calcific aortic valve disease: critical roles for matricellular, matricrine, and matrix mechanics cues. Circ Res. 2011;108:151024.

38. Benton JA, Kern HB and Anseth KS. Substrate properties influence calcification in valvular interstitial cell culture. J Heart Valve Dis. 2008;17:689-99.

39. Kloxin AM, Benton JA and Anseth KS. In situ elasticity modulation with dynamic substrates to direct cell phenotype. Biomaterials. 2010;31:1-8.

40. Zhao R, Sider KL and Simmons CA. Measurement of layer-specific mechanical properties in multilayered biomaterials by micropipette aspiration. Acta biomaterialia. 2011;7:1220-7.

41. Rabkin E, Hoerstrup SP, Aikawa M, Mayer JE, Jr. and Schoen FJ. Evolution of cell phenotype and extracellular matrix in tissue-engineered heart valves during in-vitro maturation and in-vivo remodeling. J Heart Valve Dis. 2002;11:308-14; discussion 314.

42. Hoerstrup SP, Sodian R, Daebritz S, Wang J, Bacha EA, Martin DP, Moran AM, Guleserian KJ, Sperling JS, Kaushal S, Vacanti JP, Schoen FJ and Mayer JE, Jr. Functional living trileaflet heart valves grown in vitro. Circulation. 2000;102:III44-9.

43. Merryman WD, Lukoff HD, Long RA, Engelmayr GC, Jr., Hopkins RA and Sacks MS. Synergistic effects of cyclic tension and transforming growth factor-beta1 on the aortic valve myofibroblast. Cardiovascular pathology : the official journal of the Society for Cardiovascular Pathology. 2007;16:26876.

44. Thayer $\mathrm{P}$, Balachandran $\mathrm{K}$, Rathan $\mathrm{S}$, Yap CH, Arjunon S, Jo H and Yoganathan AP. The effects of combined cyclic stretch and pressure on the aortic valve interstitial cell phenotype. Annals of biomedical engineering. 2011;39:1654-67.

45. Balachandran K, Alford PW, Wylie-Sears J, Goss JA, Grosberg A, Bischoff J, Aikawa E, Levine RA and Parker KK. Cyclic strain induces dual-mode endothelial-mesenchymal transformation of the cardiac valve. Proceedings of the National Academy of Sciences of the United States of America.

2011;108:19943-8. 


\section{Figure Legends}

Figure 1. Fabrication of VIC-laden hydrogels. A, Methacrylation of gelatin (GelMA) and hyaluronic acid (HAMA), respectively, occurs by the addition of methacrylic anhydride groups to dissolved gelatin and hyaluronic acid. B, Schematic depiction of photocrosslinking GelMA and HAMA. By dissolving HAMA and GelMA into a solution containing a photoinitiator, when exposed to UV light, the polymers form crosslinks between the methacrylate groups, creating an ECM-like mesh. C, Schematic depiction of fabricating VIC-laden hydrogels. (1) VICs are isolated from culture and resuspended into a photoinitiator solution containing dissolved $1 \%(\mathrm{w} / \mathrm{v})$ HAMA and 5\% (w/v) GelMA; (2) Cell-polymer suspension is pipetted on to a plate between two spacers $(450 \mu \mathrm{m})$ and (3) exposed to light at an intensity of $2.5 \mathrm{~mW} / \mathrm{cm}^{2}$ for 30 seconds $(\lambda=365 \mathrm{~nm})$, which yields a (4) crosslinked VIC-laden hydrogel disc. VIC-laden hydrogels are then cultured for up to 21 days.

Figure 2. Calcification potential of $3 \mathrm{D}$ in vitro valve model. A, Representative Alizarin red $\mathrm{S}$ staining of VIC-laden hydrogels cultured in control media (CM), osteogenic media (OM), or osteogenic media with $\mathrm{TNF} \alpha(\mathrm{OM}+\mathrm{TNF} \alpha)$ for $1,7,14$, and 21 days. B, Representative Alizarin red S staining and $\mathbf{C}$, Von Kossa staining of VIC-laden hydrogels cultured in CM or OM with $\mathrm{TNF} \alpha(0,1,5$ or $10 \mathrm{ng} / \mathrm{mL})$ for 21 days. Arrows indicate calcific noduli. Bar: $100 \mu \mathrm{m}$. D, Number of calcific noduli per hydrogel section. $\mathrm{N}=3$. E, Calcium mineral measured every 3 days in hydrogel constructs cultured in $\mathrm{OM}$ or $\mathrm{OM}+\mathrm{TNF} \alpha$. $\mathrm{N}=3$ per time point. **: $\mathrm{p}<0.05$. Data depicted as mean $\pm \mathrm{SD}$.

\section{Figure 3. Osteogenic environment activates quiescent VICs in 3D hydrogels. A-D,} Representative immunofluorescence $\alpha$-SMA staining (green), nuclei (blue) of a non-calcified and calcified regions of aortic heart valve of a patient with aortic stenosis. $\mathrm{N}=4$. V: Zona

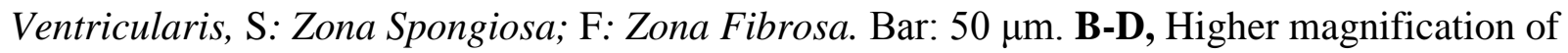
A/C; Bar: $50 \mu \mathrm{m}$. E-F, Immunofluorescence $\alpha$-SMA staining in cell-laden hydrogels cultured for 21 days in control $(\mathrm{CM})$ or osteogenic media $(\mathrm{OM})$. Bar; $50 \mu \mathrm{m}$. G, Quantification of $\alpha$-SMApositive cells per high-power field (HPF), N=3. H, $\alpha$-SMA mRNA expression. t-Test, **: p < 0.01. I, J mRNA expression of $\alpha$-SMA and Runx2 for OM or OM+TNF $\alpha$ treatments quantified at day 7, 14, and 21 and normalized to day 1 values. $\mathrm{N}=3$. Two-way ANOVA, ***: $\mathrm{p}=0.001$, * $\mathrm{p}=0.03$. Data depicted as mean \pm SD. K-M, After 21 days in OM culture, VICs immuno- 
positive for $\mathbf{K}$, both $\alpha$-SMA and Runx2, L, only cytosolic Runx2, and M, nuclear Runx 2 were observed. Bars: $10 \mu \mathrm{m}$.

Figure 4. Silencing $\boldsymbol{\alpha}$-SMA reduces osteogenesis. VIC-laden hydrogels were cultured up to 21 days in control media (CM), osteogenic media (OM), or osteogenic media with TNF $(\mathrm{OM}+$ TNFa). Silencing of $\alpha$-SMA (Si- $\alpha \mathrm{SMA}$ ) was performed twice per week. Scramble (SCR) SiRNA served as control. A, $\alpha$-SMA mRNA expression. B, Runx2 mRNA expression. C, Calcium content normalized for DNA content at day 21. D, Alizarin red S staining of VIC-laden hydrogels. Data depicted as mean $\pm \mathrm{SD}, *: \mathrm{p}<0.05$.

Figure 5. Analysis of VIC phenotypes in human CAVD. A, Representative immunofluorescent image for Runx 2 and $\alpha$-SMA with DAPI stained nuclei of calcified aortic heart valve. Bar: $100 \mu \mathrm{m}$. B, Quantification of $\alpha$-SMA and Runx2 immunofluorescence (shown as arbitrary units $(\mathrm{AU}))$ for every $(\mathrm{n}=\ldots .$.$) DAPI-positive cell in 21$ different images collected from histological sections of CAVD tissues from $n=4$ human donors. C, Fraction of Runx 2 nuclear translocation for each cell analyzed in B. Within the tissue sections VICs immunopositive for $\mathbf{D}, \alpha$-SMA only, E, both $\alpha$-SMA and Runx2, F, only cytosolic Runx2, and $\mathbf{G}$, mostly nuclear Runx 2 were observed. Bars: $5 \mu \mathrm{m}$.

Figure 6. Schematic illustration of simulating progression of VIC phenotypes in CAVD. Pathophysiological cellular concept of "active" mineralization in CAVD. Quiescent VICs (qVICs) become activated myofibroblast-like VICs (aVICs) hallmarked by increased $\alpha$-SMA expression. In healthy valves, cells may return to a quiescent state, but in CAVD, aVICs can differentiate into osteoblast-like VICs (oVICs) characterized by decreased $\alpha$-SMA expression and increased Runx2 expression. Using a 3D culture platform, the entire cellular driven disease process of CAVD can be modeled. 
Figure 1

A
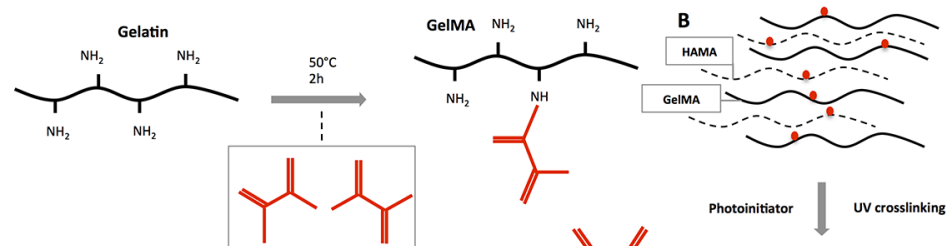

Hyaluronic Acid

Methacrylic anhydride
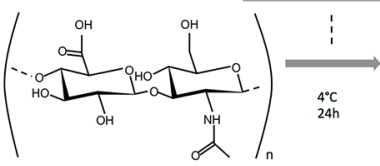

$4^{\circ} \mathrm{C}$

$24 \mathrm{~h}$

C

Light $(\lambda=365 \mathrm{~nm}$ )
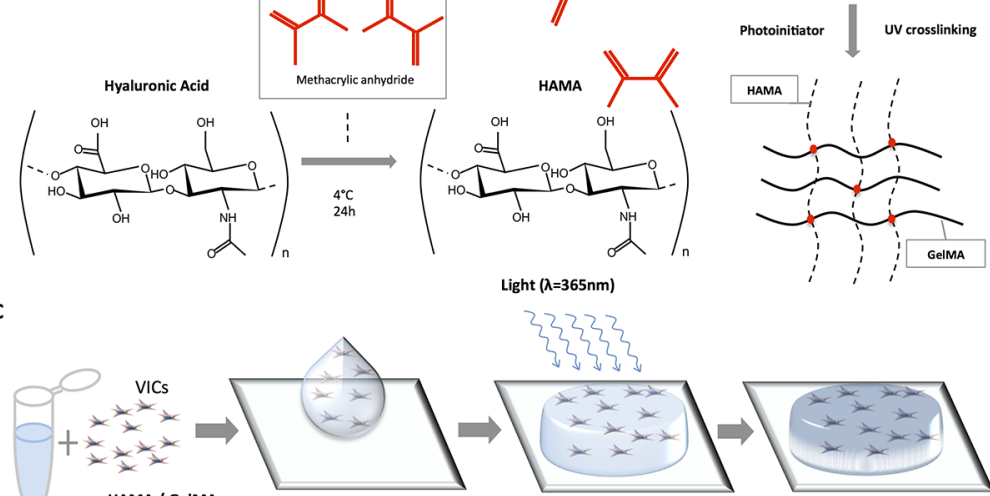

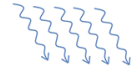

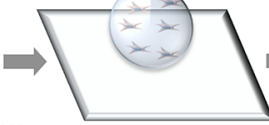

in $\mathrm{PI}(0.1 \%)$ solution 
Figure 2
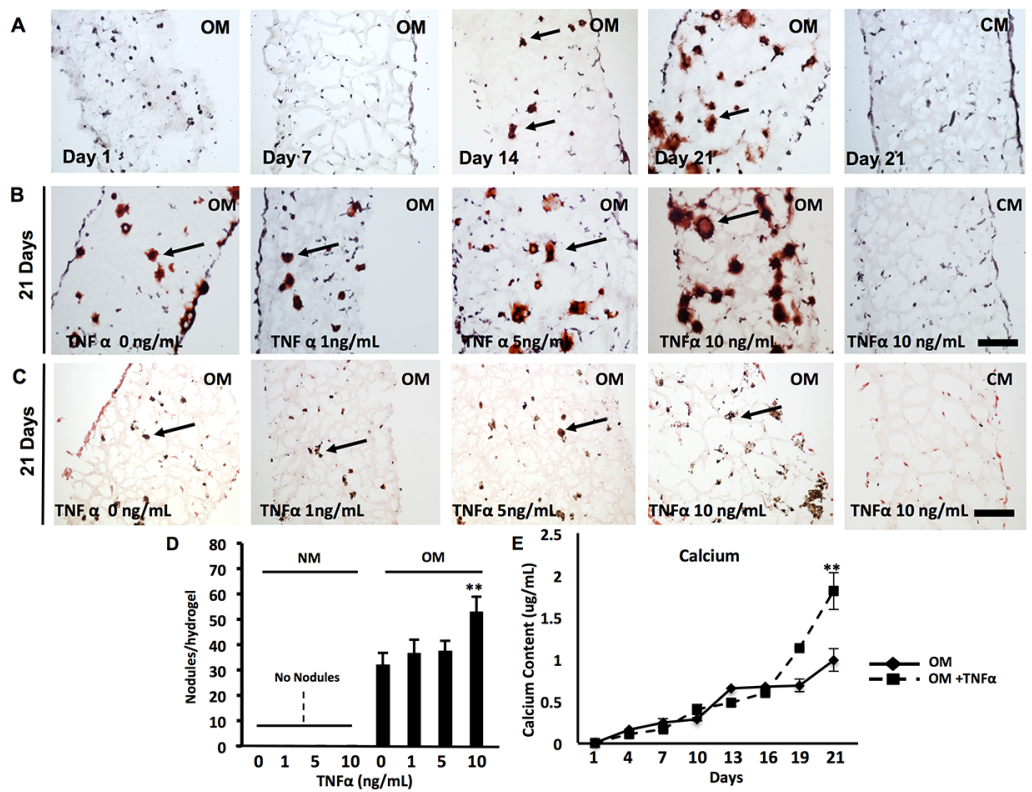


\section{Figure 3}

Human Aortic Valve Tissue
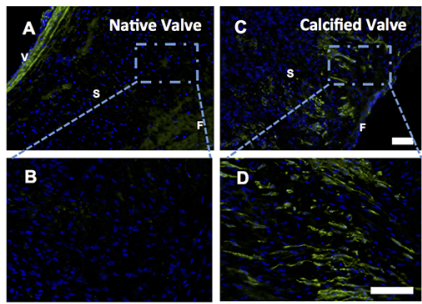

I

$\alpha$-SMA expression
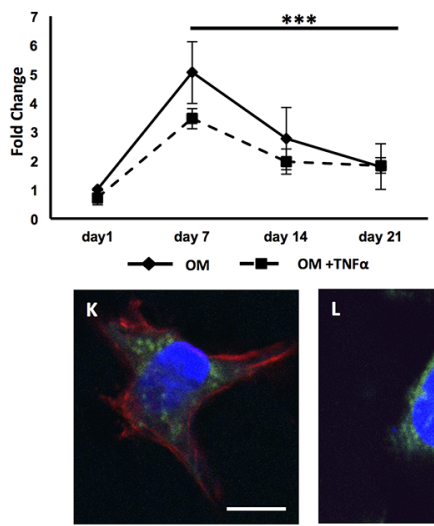

3D Hydrogel
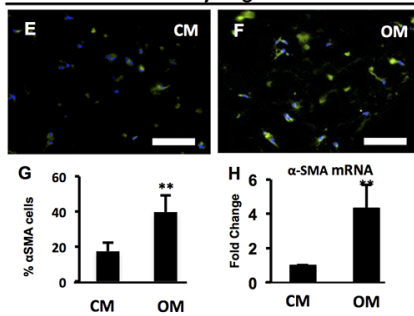

J

Runx2 expression

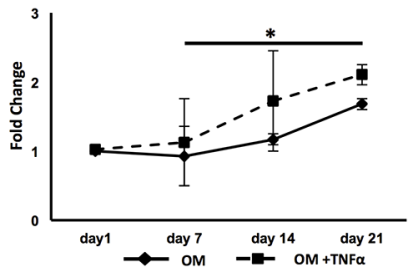

M 
Figure 4

A $\alpha$-SMA mRNA at day 21

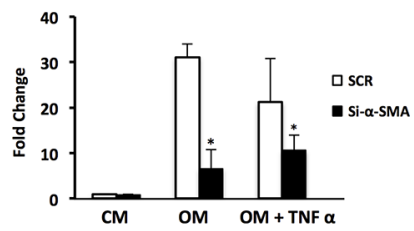

B
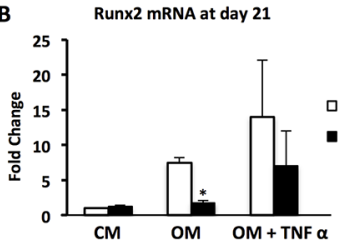

C

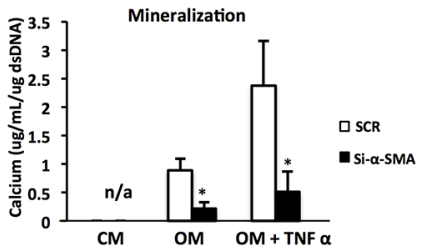

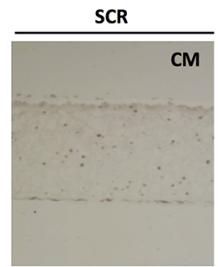
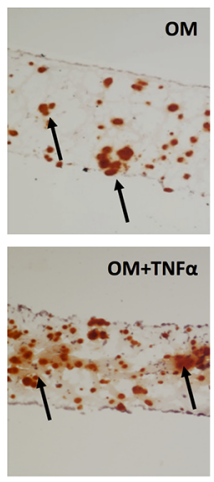

Si- $\alpha$-SMA

CM

OM

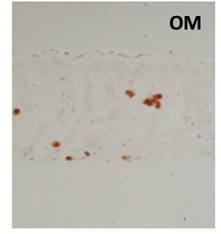

OM+TNF $\alpha$

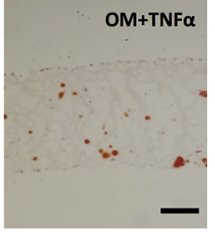


Figure 5
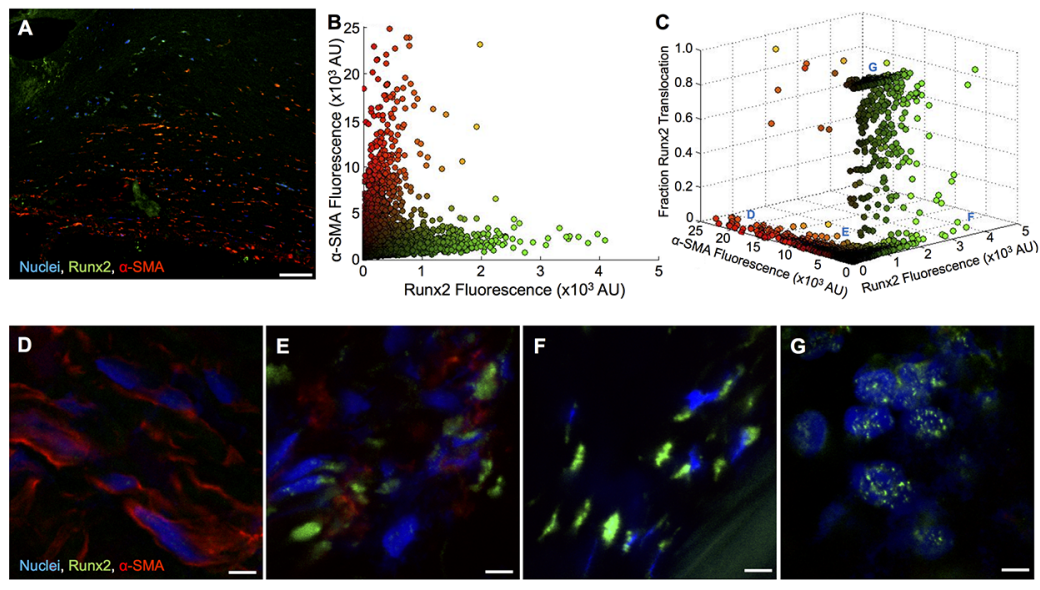
Figure 6

\section{CAVD}

Quiescence

Activation

Calcification
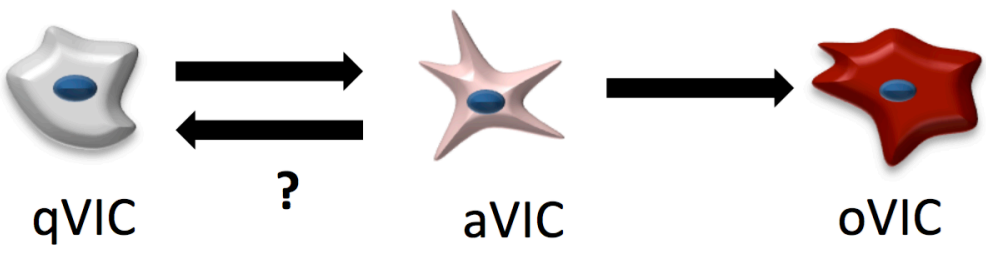

aVIC

oVIC

$\alpha$-SMA (-)

$\alpha-S M A \uparrow$

$\alpha-S M A \downarrow$

Runx2 